\title{
Controlling the order of triblock copolymer via confinement induced by forced self-assembly
}

\author{
Sébastien Roland*, Guillaume Miquelard-Garnier, Matthieu Gervais, Alain Guinault, \\ Cyrille Sollogoub*
}

PIMM-UMR 8006, ENSAM, CNRS, CNAM, 151 bvd de l'Hôpital, 75013 Paris, France

\begin{abstract}
A B S T R A C T
We report the making of multilayered self-assembled films by coextrusion, composed of alternated layers of confining polycarbonate and confined poly(styrene-b-butadiene- $b$-methyl methacrylate), whose blocks are chemically different from the confining polymer, and presenting a self-assembled morphology directly after extrusion. The triblock copolymer layers thicknesses was varied from few hundreds to few tens of nanometers. As the triblock layer thickness is decreased and the draw ratio is increased, the triblock morphology is constrained into a preferential orientation and higher long-range order is observed by transmission electron microscopy and small angle X-ray scattering. This one-step and industrially scalable method allowing long-range ordering of the nanodomains is of interest for many engineering applications for which producing large quantity of materials is necessary.
\end{abstract}

\section{Introduction}

Block copolymers have received much interest in the past decades due to their ability to self-assemble with well-defined phase separation at the nanometer scale that leads to domains of the size of few tens of nanometers, which make them very attractive for many applications (e.g., in the field of nanoelectronics, nanolithography, biosensors, optoelectronics, membranes, holographic gratings, etc.) [1-3]. These engineering applications demand control over the orientation and the position of the nanodomains. However, during the self-assembling process, nanodomains nucleate randomly and grow as a polygrain texture with a periodical order maintained only over few micrometers. To overcome this lack of order at the macroscale and induce nanodomain orientation and ordering, external stimuli have been successfully used. Several methods, such as solvent or temperature annealing sometimes combined with shearing, are commonly employed efficiently to create long-range ordering in thin films or in the bulk $[1,4,5]$.

Multilayer coextrusion is an attractive technique to produce films made of thousands of alternating layers with individual thickness down to $\sim 10 \mathrm{~nm}$. In pioneering work, Baer and coworkers used what they have termed a "forced assembly" technique to combine

\footnotetext{
* Corresponding authors.

E-mail addresses: sebastien.roland@ensam.eu (S. Roland), cyrille.sollogoub@cnam.fr (C. Sollogoub).
}

immiscible polymer pairs into unique multilayer structures, leading to remarkable properties such as gas barrier, mechanical, and optical properties [6-9]. Recently, this forced assembly technique has been used by Korley et al. to confine spherical and cylindrical ABA triblock copolymer [10-12]. These studies mainly focus on the relationship between the nanostructure and the mechanical properties of the resulting films, while the extrusion was carried out at a temperature below the order-disorder transition temperature $\left(T_{\mathrm{ODT}}\right)$. Flow orientation and confinement induced by multilayer coextrusion creates long-range ordering of cylinders in the extrusion direction. The best ordering has been obtained for the thinnest layers after annealing (performed at a temperature above $T_{\mathrm{g}}$ of the major block and below $T_{\mathrm{ODT}}$ ).

Because of the three chemically different blocks, ABC triblock terpolymers can lead to a larger set of microstructure morphologies and allows the creation of original patterns [13-15]. Theoretically, $\mathrm{ABC}$ copolymers can develop tens of morphologies that are usually studied via computer calculations, by taking into account the $\chi$ parameters between the three blocks [16-18]. Experimentally, poly(styrene- $b$-butadiene- $b$-methyl methacrylate) (SBM) morphologies were thoroughly studied by Stadler et al.: lamellar, knitting, dotted-cylinders, cylinder-within-cylinders, spheres-onspheres morphologies or other morphologies peculiar to $A B C$ copolymers have been successfully observed [19-22].

In this paper, we describe a direct and scalable method to produce large quantities of a hierarchical material presenting a double organization of the matter at the nano- and the microscale, by 
using the coextrusion multilayer process, without additional thermal annealing, to confine and self-assemble SBM. We study the influence of the interfaces on the self-assembling process by using a confining polymer having no particular compatibility with the copolymer blocks. In Korley's work, the impact of the draw ratio on the orientation of the morphology has not been clearly addressed. This contribution helps to demonstrate that processing parameters, especially the draw ratio, which has a direct impact on the confining thickness, are important in the improvement of the ordering mechanism of the SBM triblock copolymer morphology.

\section{Materials and methods}

\subsection{Materials}

Polycarbonate 121R (PC) was purchased from Sabic and used as received (confining polymer). Its melt flow index is $17.5 \mathrm{~g} / 10 \mathrm{~min}\left(300^{\circ} \mathrm{C} / 1.2 \mathrm{~kg}\right)$, as provided by Sabic. Its glass transition temperature is $153^{\circ} \mathrm{C}$ (measured by Differential Mechanical Thermo-Analysis [Q800, TA Instruments], at a rate of $1^{\circ} \mathrm{C} / \mathrm{min}$ and at a strain of $0.1 \%)$. Poly(styrene- $b$-butadiene- $b$-methyl methacrylate) E20 (SBM, PS-PB-PMMA) triblock copolymer was gratefully supplied by Arkema and used as received without further purification.

\subsection{Gel permeation chromatography}

Polymer molecular weights were determined by GPC at $40^{\circ} \mathrm{C}$ using THF as eluent on a Waters apparatus equipped with three Styragel columns HR0.5, HR3 and HR4 and with a Waters 2414 refractive index detector at an elution rate of $1 \mathrm{~mL} / \mathrm{min}$. Polystyrene were used as standards. GPC curves show that a large fraction of diblock (SB) remains in the triblock copolymer. Deconvolution of the SBM and SB curves using a Gaussian distribution for both polymers $\left(r^{2}=0.99\right)$ leads to $M_{n}=100 \mathrm{~kg} / \mathrm{mol}(\mathrm{PDI}=1.1)$ for the triblock, and $M_{n}=50 \mathrm{~kg} / \mathrm{mol}(\mathrm{PDI}=1.05)$ for the diblock. The calculation of their area, assuming the two polymers have the same refractive index, leads to $55 \mathrm{~mol} \%$ of SBM and $45 \mathrm{~mol} \%$ of SB in the sample as provided by Arkema (see Fig. SI-1). The PC $M_{\mathrm{W}}$ was also measured by GPC, and is $40 \mathrm{~kg} / \mathrm{mol}(\mathrm{PDI}=2.2)$.

\section{3. ${ }^{1} H N M R$}

${ }^{1} \mathrm{H}$ NMR spectra were recorded with 32 scans on a Bruker apparatus at $300 \mathrm{MHz}$. Fig. SI-2 displays the chemical shift in ppm from tetramethylsilane with the solvent as an internal indicator $\left(\mathrm{CDCl}_{3} ; 7.26 \mathrm{ppm}\right)$, and integration of the main peaks. The calculation of the molar ratio of the three components of the triblock is presented in Supporting information. This gives for the whole sample (SB and SBM) a molar composition of $26 \%$ for PS, $47 \%$ for PB and 27\% for PMMA. In wt\%, this leads to $34 \%, 32 \%$ and $34 \%$ for PS, PB and PMMA respectively, in very good agreement with the supplier information (33-33-33wt\%). Combining NMR and GPC results, one can conclude that the composition of the triblock with $M_{n}=100 \mathrm{~kg} / \mathrm{mol}$ is close to $25 \mathrm{~kg} / \mathrm{mol}, 25 \mathrm{~kg} / \mathrm{mol}$, and $50 \mathrm{~kg} / \mathrm{mol}$ for the PS, PB and PMMA blocks respectively while the diblock is $25 \mathrm{~kg} / \mathrm{mol}$ and $25 \mathrm{~kg} / \mathrm{mol}$ for PS and PB. In consequence, the composition of triblock itself is actually close to $25-25-50$ (wt\%).

\subsection{Rheology}

To determine the $T_{\mathrm{ODT}}$ and the morphology of the triblock copolymer near the processing temperature, melt rheology was performed on an Anton-Paar rheometer using $25 \mathrm{~mm}$ coaxial disks, separated by a $1 \mathrm{~mm}$ gap. Complex viscosity (see Fig. SI-3), storage modulus $G^{\prime}$ and loss modulus $G^{\prime \prime}$ were recorded in a frequency range from $10^{-2}$ to $10^{2} \mathrm{~Hz}$ at various temperatures. The sample was tested isothermally in a nitrogen atmosphere. A strain amplitude of $1 \%$ was used to keep the response in the linear domain.

\subsection{Films preparation}

Prior to extrusion, SBM and PC pellets were dried in a SOMOS dry air dryer T 20 eco system, respectively at $80^{\circ} \mathrm{C}$ for $3 \mathrm{~h}$ and $120^{\circ} \mathrm{C}$ for $4 \mathrm{~h}$. The PC grade was chosen so that the viscosity of the two polymers matches at the processing temperature $\left(240^{\circ} \mathrm{C}\right)$ and shear rate $\left(5-20 \mathrm{~s}^{-1}\right)$. In this range of shear rates, assumed to be those in the multiplying elements, the viscosity ratio lies between 0.5 and 2 , as measured by rheology (Fig. SI-3). Films were prepared using multiplying elements at the end of a classical trilayer coextrusion set-up with SBM at the center of the trilayer flow. The processing route consists of two single screw extruders, a 3-layer coextrusion feedblock (ABA), a series of layer-multiplying die elements, an exit film die, and a thermally regulated chill roll. A $30 \mathrm{~mm}$-diameter Mapre extruder and a $20 \mathrm{~mm}$-diameter Scamex extruder were used for the PC and the SBM, respectively. The amount of each polymer in the film was set to $90 \%$ PC for $10 \%$ SBM (wt\%) by adjusting the screw speeds of each extruder to control the throughput. The triblock was extruded at $240{ }^{\circ} \mathrm{C}$ and the $\mathrm{PC}$ at $300^{\circ} \mathrm{C}$, the multiplying elements block being set at $240^{\circ} \mathrm{C}$. The theoretical number of the alternating layers $(\mathrm{N})$ in the film is directly determined by the number of elements $(n)$ through the equation $\mathrm{N}=2^{(n+1)}+1$. Depending on the targeted thickness of the confined SBM layer, 7 (257 total layers) or 9 (1025 layers) multiplying elements were used. After the flat die, the films were drawn with a chill roll at $120^{\circ} \mathrm{C}$. Draw ratio (Dr) can be defined as the ratio of the extrusion flow rate at the die over the draw rate of the chill roll and is varied from 1 to 11 (draw rate ranging from $90 \mathrm{~cm} / \mathrm{min}$ to $1000 \mathrm{~cm} / \mathrm{min}$ ).

\subsection{Transmission electron microscopy}

The morphology of the SBM block copolymer within the extruded films was studied by TEM in the out-of-plane and the transverse directions, so that the electron beam is parallel and perpendicular to the extrusion direction, respectively. TEM micrographs were taken on a Zeiss 912 operated at an accelerated voltage of $80 \mathrm{kV}$ and equipped with a Veleta camera (Olympus). In a first step, pre-faced pieces of the extruded films were cut and immersed in a $1 \%$ aqueous $\mathrm{OsO}_{4}$ solution (Electron Microscopy Sciences) for $48 \mathrm{~h}$. Osmium tetroxide stains preferentially unsaturated carboncarbon double bonds and gives to the PB regions the darkest colors in TEM observations. The PS regions are also stained but the $\mathrm{C}-\mathrm{C}$ bonds contained in the styrene ring being less reactive than the unsaturated rubber, they appears brighter. Finally, PMMA and PC are the least reactive to $\mathrm{OsO}_{4}$. In consequence, they appear transparent, thus brighter in the images. The cross-linking of the $\mathrm{PB}$ rubbery phase during the staining causes hardening and allows the ultramicrotomy to be performed at room temperature [23]. The stained films were then ultramicrotomed into slices of approximately 70-80 nm thick, using an LKB Ultrotome V 2088 equipped with a Diatome diamond knife.

\subsection{Small angle X-ray scattering}

SAXS measurements were recorded using a Xerocs X-Ray system. $\mathrm{CuK} \alpha$ lab source with $\lambda=1.54 \AA$ 少as generated by a tungsten filament operated at $50 \mathrm{kV}$ and $1 \mathrm{~mA}$. The beam was collimated by two pairs of tungsten blades mounted at right angles to each other (beam size of $0.8 \times 0.8 \mathrm{~mm}^{2}$ ) and the sample was placed at $1215 \mathrm{~mm}$ from the detector. The scattering vector $q$, defined as $q=4 \pi \sin \theta / \lambda$ (where $\theta$ is half the scattering angle), ranged from $10^{-2}$ to $0.7 \AA^{-1}$ 

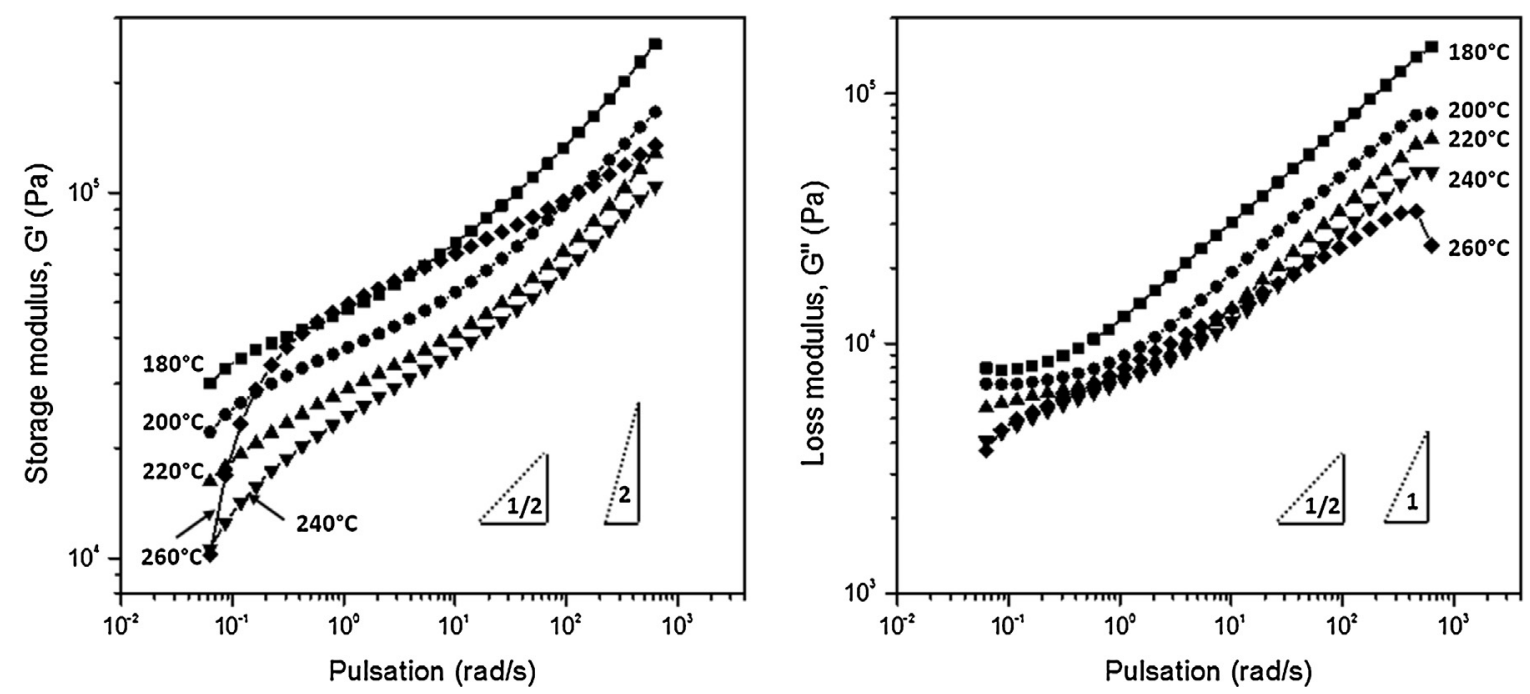

Fig. 1. Dynamic storage $G^{\prime}$ and loss $G^{\prime \prime}$ moduli of SBM E20 pellets as a function of pulsation at different temperatures indicated on the figure.

and was calibrated using a silver behenate standard sample. Fit-2D analysis software was used to integrate the scattering patterns.

\section{Results and discussion}

Fig. 1 presents the dynamic storage $G^{\prime}$ and loss moduli $G^{\prime \prime}$ of the triblock copolymer SBM E20 pellets at different temperatures. Looking at the storage modulus, the relaxation spectrum shows a time-dependent component at low frequency indicating a selfassembling morphology [24-26]. Normally, for a disordered state, the slope of the storage modulus should be the same as that of a homopolymer melt in the terminal region $\left(\mathrm{G}^{\prime} \propto \omega^{2}\right)[27,28]$. In contrast, the slope for both $G^{\prime}$ and $G^{\prime \prime}$ is close to 0.5 for temperatures between 180 and $240^{\circ} \mathrm{C}$. These slopes were attributed to a lamellar morphology for diblock copolymers [29] as well as for SBM triblock, according to Di Cola et al. [30] It should be noted that $\mathrm{G}^{\prime}$ at $260^{\circ} \mathrm{C}$ shows an increase compared to the other curves which can be attributed to the degradation of the SBM due to a cross-linking of the $\mathrm{PB}$ phase during the analysis, which is not observed at the processing temperature $\left(240^{\circ} \mathrm{C}\right)$. These experiments reveal that, during extrusion, the block copolymer is still self-assembled and does not flow as a homopolymer since the elastic contribution $\left(G^{\prime}\right)$ of the dynamic modulus is higher than the viscous one $\left(\mathrm{G}^{\prime \prime}\right)$.

Fig. 2 presents the scheme of the coextrusion process and a typical TEM image cross-section of the multilayered PC/SBM films obtained in this study. Knowing the difficulty to coextrude polymers with mismatched rheological behavior, we may expect interfacial distortion and/or layer breakups when coextruding self-assembled block copolymer below its $T_{\mathrm{ODT}}\left(\mathrm{G}^{\prime}>\mathrm{G}^{\prime \prime}\right)$ with a thermoplastic $\left(G^{\prime \prime}>G^{\prime}\right)[31]$. However, as can be seen in Fig. 2, the obtained multilayered films of PC and SBM are stable and present continuous layers with slight variations in SBM thicknesses. This stability could be explained by the fact that both storage moduli and viscosities of PC and SBM are actually in the same range at the extrusion shear rate $\left(5-20 s^{-1}\right)$ (see Fig. SI-3). Thanks to the distribution of the SBM layers thicknesses at a given experimental condition, it is possible to study the effect of the draw ratio on the morphology at a given thickness.

Fig. 3 shows TEM micrographs of the confined SBM layers crosssections as a function of thickness at $\mathrm{Dr}=2, \mathrm{Dr}=8$, and $\mathrm{Dr}=11$. It also shows the transverse direction of films made at $\mathrm{Dr}=11$. The periodical thickness of the block copolymer morphology appears to be around $50 \mathrm{~nm}$, which is quite similar to what has been previously reported in the literature for such material $[19,20]$. As the confined layer thickness decreases from 200 to $100 \mathrm{~nm}$, the number of block copolymer periods in the confined layer decreases from 4 to 2 . At low $\mathrm{Dr}$, the morphology seems to be locally disordered, even though one can guess a global orientation of the morphology along the interfaces. Qualitatively, a better organization of the nanodomains is seen at high Dr, but also for decreasing layer thicknesses.

The morphology of the triblock copolymer is hard to determine with the TEM micrographs. It seems that both lamellae (PS
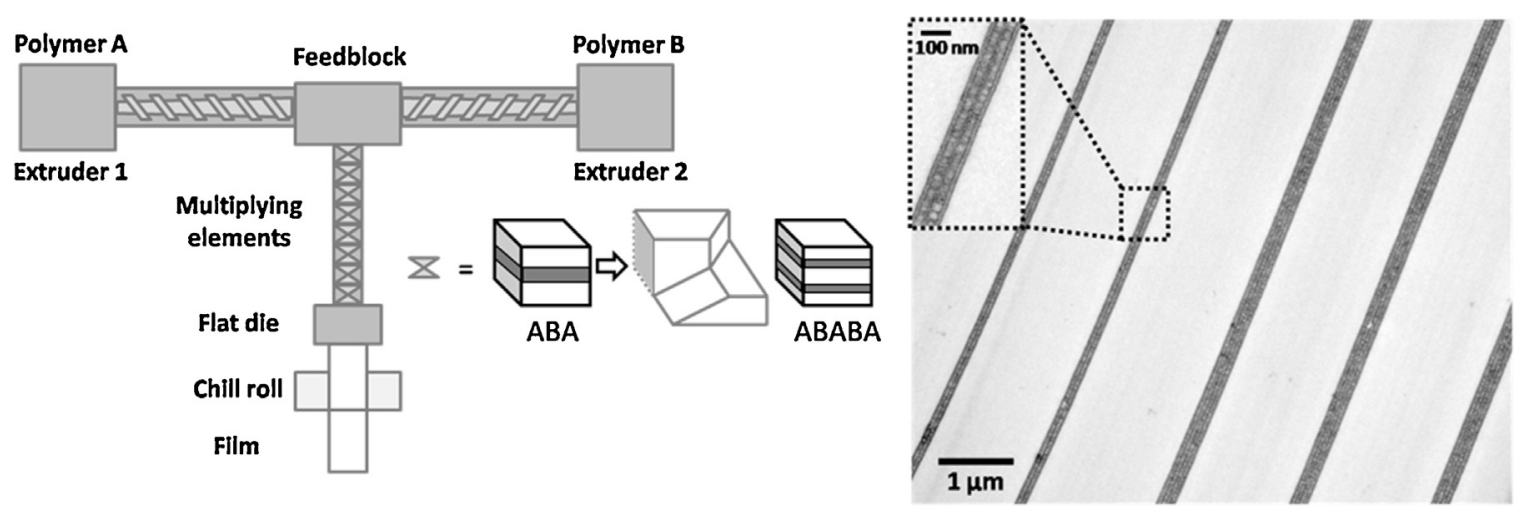

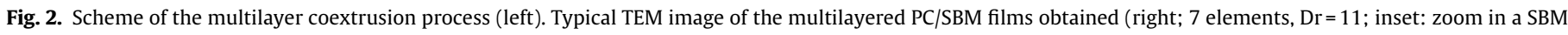
layer). 


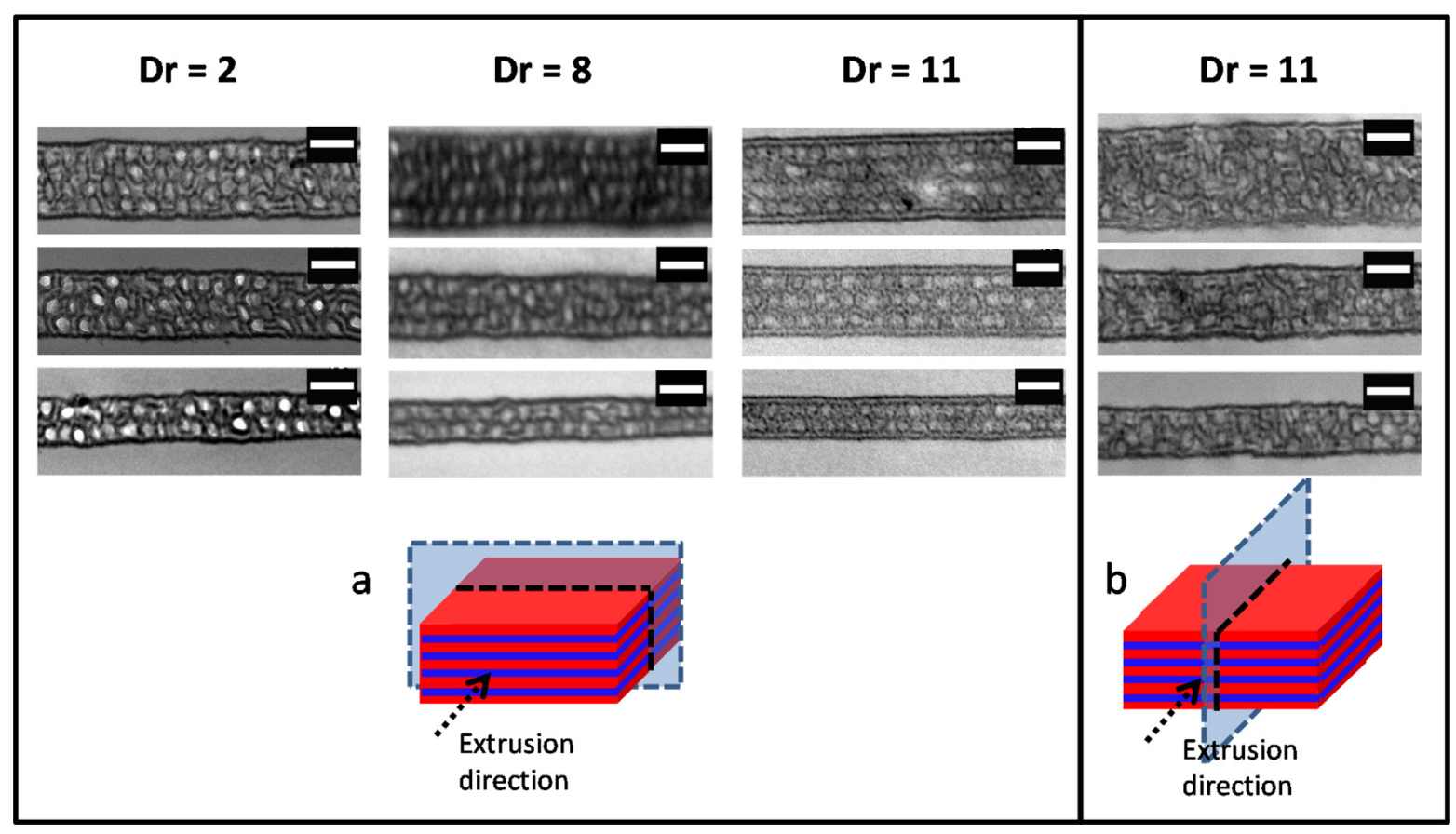

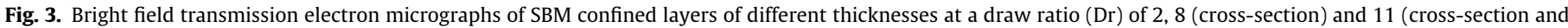
transverse). Scale bars $=100 \mathrm{~nm}$. The schemes illustrate the film section observation: cross-section (a) and transverse (b).

and $\mathrm{PB}$ ) and cylinders (or nodules, PMMA) are observed in Fig. 3. The presence of SB diblock copolymer in the melt could lead to a mixed and therefore complex morphology. At these compositions, both copolymers (SB and SBM) should have a lamellar morphology $[32,33]$. However, in a blend, the SB diblock copolymer will be located at the S-B interface, increasing the number of junction points to this interface. To avoid the entropy loss due to the chain stretching, the interfacial area increases compared to the $\mathrm{B}-\mathrm{M}$ interface. The consequence is the curvature of the S-B interface, which is inexistent for pure lamellar SBM triblock copolymer. Cylinders-in-lamellae, lamellae with fluctuations or double gyroid can then be observed with slight blend composition variations $[32,33]$. It is noteworthy that the core-shell cylinders and doublegyroid morphologies identified in Ref. [33] by TEM for a 48/52 blend composition of SBM/SB resemble to our TEM observations. The PCSBM interfaces influence the alignment of the morphology: parallel lamellae clearly appear at the interface due to a preferential wetting towards the polycarbonate layers (see Fig. 3).

Besides, at high draw ratio, the lamellar structure at the interface is more pronounced and within the layer, the arrangement of the morphology appears also more regular even for thicker layers.
When probing the transverse direction, it appears that fewer nodules are observed in this direction and that the morphology is less ordered. It can also be noticed that the interface is less stable, showing some fluctuations that are not seen in the cross-section image, which can be attributed to flow fluctuations during extrusion.

At the interface between the PC layer and the SBM, a black region can be observed and attributed to the PB phase. This black region takes the shape of a well-defined line at high draw ratios, perfectly parallel to the interface. To explain the interface in the case of PC/SBM multilayered films, the solubility parameters were used to calculate the Flory-Huggins parameters $\chi$ (Table 1 ) of each polymer pairs by using the approach based on Hildebrand solubility parameters:

$$
\chi=\frac{V_{\mathrm{AB}}}{\mathrm{RT}}\left(\delta_{\mathrm{A}}-\delta_{\mathrm{B}}\right)^{2}
$$

Where $V_{\mathrm{AB}}$ is the geometric mean of molar volumes of polymer $\mathrm{A}$ and polymer $\mathrm{B}, R$ is the universal gas constant $(8.314 \mathrm{~J} / \mathrm{K} / \mathrm{mol}), T$ is the processing temperature, $\delta_{\mathrm{A}}$ and $\delta_{\mathrm{B}}$ are the solubility parameters of polymer A and polymer B, respectively.

Table 1

Molar volumes, solubility parameters, and $\chi$ parameters of the PC/SBM system.

\begin{tabular}{|c|c|c|c|c|}
\hline Polymer pair & $\begin{array}{l}\text { Geometric mean } \\
\text { of molar volumes, } \sqrt{V_{\mathrm{A}} V_{\mathrm{B}}} \text {, } \\
\mathrm{cm}^{3} / \mathrm{mol}^{\mathrm{a}}\end{array}$ & $\begin{array}{l}\text { Cohesive } \\
\text { energy density }\left(\delta_{\mathrm{A}}-\delta_{\mathrm{B}}\right)^{2}, \mathrm{~J} / \mathrm{cm}^{3 \mathrm{~b}}\end{array}$ & $\begin{array}{l}\chi_{\mathrm{AB}} \text { using Eq. }(1) \\
\left(240^{\circ} \mathrm{C}\right)^{\mathrm{c}}\end{array}$ & $\begin{array}{l}\chi_{\mathrm{AB}} \text { using Ref. [34] } \\
\left(240^{\circ} \mathrm{C}\right)^{\mathrm{d}}\end{array}$ \\
\hline PC/PMMA & 122.9 & 0.08 & 0.0023 & 0.016 \\
\hline $\mathrm{PC} / \mathrm{PS}$ & 130.8 & 1.00 & 0.029 & 0.294 \\
\hline $\mathrm{PC} / \mathrm{PB}$ & 102.9 & 7.40 & 0.213 & 0.856 \\
\hline PS/PMMA & 92.1 & 1.64 & 0.047 & 0.119 \\
\hline $\mathrm{PS} / \mathrm{PB}$ & 77.1 & 2.96 & 0.085 & 0.149 \\
\hline PMMA/PB & 72.5 & 9.00 & 0.259 & 0.460 \\
\hline
\end{tabular}

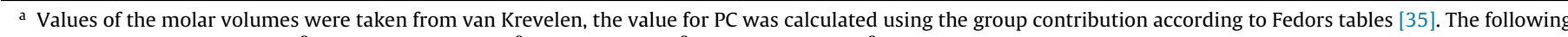
values were used: $V_{\mathrm{PC}}=174.6 \mathrm{~cm}^{3} / \mathrm{mol} ; V_{\mathrm{PMMA}}=86.5 \mathrm{~cm}^{3} / \mathrm{mol} ; V_{\mathrm{PS}}=98 \mathrm{~cm}^{3} / \mathrm{mol} ; V_{\mathrm{PB}}=60.7 \mathrm{~cm}^{3} / \mathrm{mol}$.

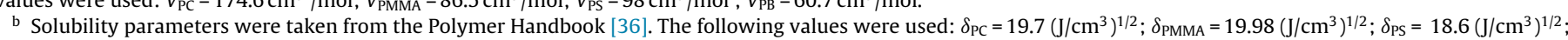
$\delta_{\mathrm{PB}}=16.98\left(\mathrm{~J} / \mathrm{cm}^{3}\right)^{1 / 2}$.

c Calculated using Eq. (1).

d Calculated from the interpolation of computed values of $\chi$ at $25^{\circ} \mathrm{C}$ and $300{ }^{\circ} \mathrm{C}$ [34]. 


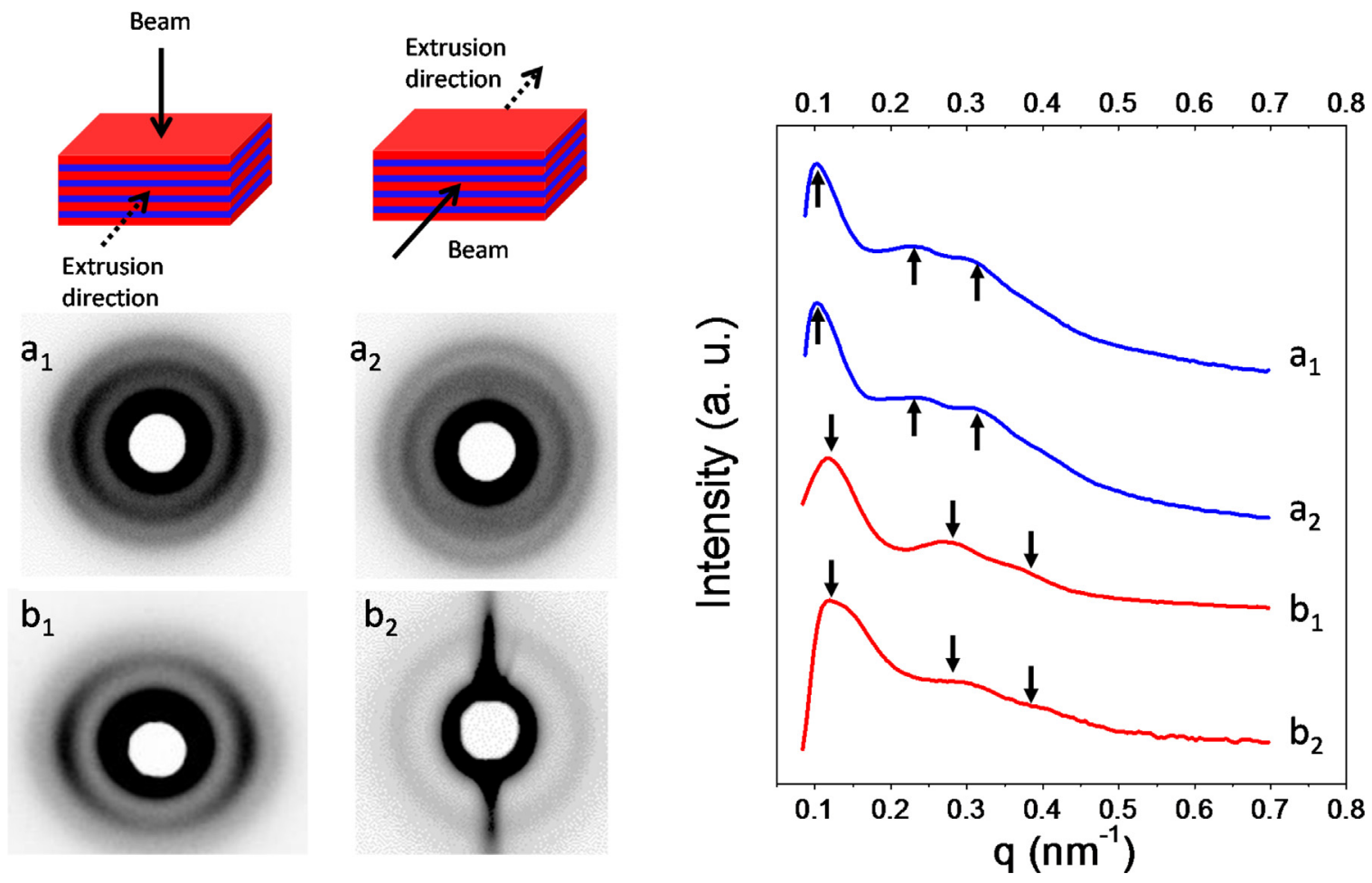

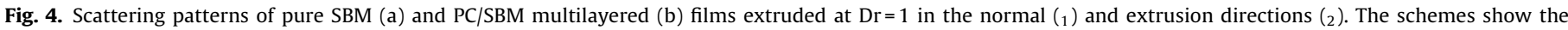

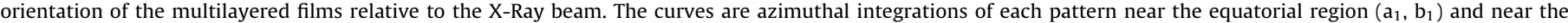
meridional region $\left(a_{2}, b_{2}\right)$. Arrows indicate the position of the peaks. Downward and upward arrows indicate the same position.

To compare the affinity of the different polymer pairs, we used experimental and calculated values for Flory Huggins and solubility parameters.

From this approach, it appears the PB phase has the smallest affinity with PC (see Table 1 ). In contrast, PMMA is most likely the block in contact with the $\mathrm{PC}$ layer. Even though $\mathrm{OsO}_{4}$ staining does not allow the contrast between PC and PMMA to be seen, it is then reasonable to assume a PC-PMMA interface. The well-aligned PB layer can then be the consequence of the PMMA "adaptive layer", which accommodate the interface, as observed for block copolymer thin films $[37,38]$.

SAXS experiments were performed on both the normal and the extrusion directions of the films to evaluate more quantitatively the degree of organization of the triblock copolymer morphology under confinement (Fig. 4) and, especially, the effect of the draw ratio (Fig. 5). In Fig. 4, the SAXS patterns of the pure SBM film (unconfined) extruded at $\mathrm{Dr}=1$ (unstretched) shows three rings in both normal and extrusion directions due to random orientation of the morphology, one of them being slightly transformed into an ellipse (Fig. $4 a_{1}$ and $4 a_{2}$ ). This is indicative of a slight orientation of the nanodomains in the extrusion direction due to the flow. As for the morphology interpretation with the SAXS patterns, if we make the assumption that the diblock copolymer is fully blended to the triblock copolymer, the blend could give rise to a double gyroid morphology [33]. This kind of morphology can be very difficult to interpret by SAXS, as reported by Hückstadt and coworkers. It is noteworthy that their results observed for a poly(styrene- $b$ 1,2 butadiene- $b$-vinylpyridine) resemble to what we observe in our case (large peaks with no particular periodicity in the peak position) [39].

For the multilayered films, the extrusion and the normal directions were also studied and SAXS patterns along with azimuthal integrations are shown in Fig. 4. Concerning the confined but unstretched samples (Fig. $4 \mathrm{~b}_{1}-\mathrm{b}_{2}$ ), a more pronounced ellipsoidal shape is observed in every direction, revealing a more oriented morphology probably due to the confinement induced by the multiple interfaces. The slight shift of the peaks towards higher $q$ values $\left(0.11-0.12 \mathrm{~nm}^{-1}\right.$, corresponding to scattering domain sizes of $57 \mathrm{~nm}$ and $52 \mathrm{~nm}$, respectively, using $q^{*}=2 \pi / d_{0}$ ) indicates a smaller scattering dimension, probably caused by a morphology change at the interface between the PC and SBM layers.

Fig. 5 compares scattering patterns of films drawn at $\mathrm{Dr}=1$ and $\mathrm{Dr}=8$ to show the influence of the draw ratio on the organization of the morphology. As the draw ratio is increased, in the normal orientation, the ellipsoidal shape becomes even clearer with its longer axis being more intense along the equator. This can be attributed to the orientation of the morphology induced by stretching during drawing. This behavior resembles to what was observed for cylindrical block copolymer being unidirectionally stretched $[40,41]$. For curve $b_{1}$, the first peak is also at $0.12 \mathrm{~nm}^{-1}$, corresponding to a scattering domain size of $52 \mathrm{~nm}$. This value is very close to the block copolymer period observed by TEM. A slight shift is also observed for the extrusion direction (curves $b_{2}$ and $b_{3}$ ) and can be attributed to a squeeze or a stretching of the morphology $\left(q^{*}=0.14 \mathrm{~nm}^{-1}, d_{0}=\right.$ $44 \mathrm{~nm}$ ). A scattering pattern along the meridional axis is observed for both the extrusion and the transverse directions and the integration shows $q^{*}, 2 q^{*}$, and $3 q^{*}$ peaks, which is characteristic of a lamellar morphology. At low Dr, the 2 nd and 3rd order are not very intense but becomes more intense at higher Dr, which indicates a better alignment of the lamellae in the stretched film and confirms the observations made by TEM. This ordering is then due to a combination of the stretching and the greater influence of the interfaces of the confining layers. For the layered films oriented in the transverse and the extrusion directions, a strong meridional streak can be observed in the scattering patterns. This could arise from the interfaces between the PC and the SBM layers, as it was observed 


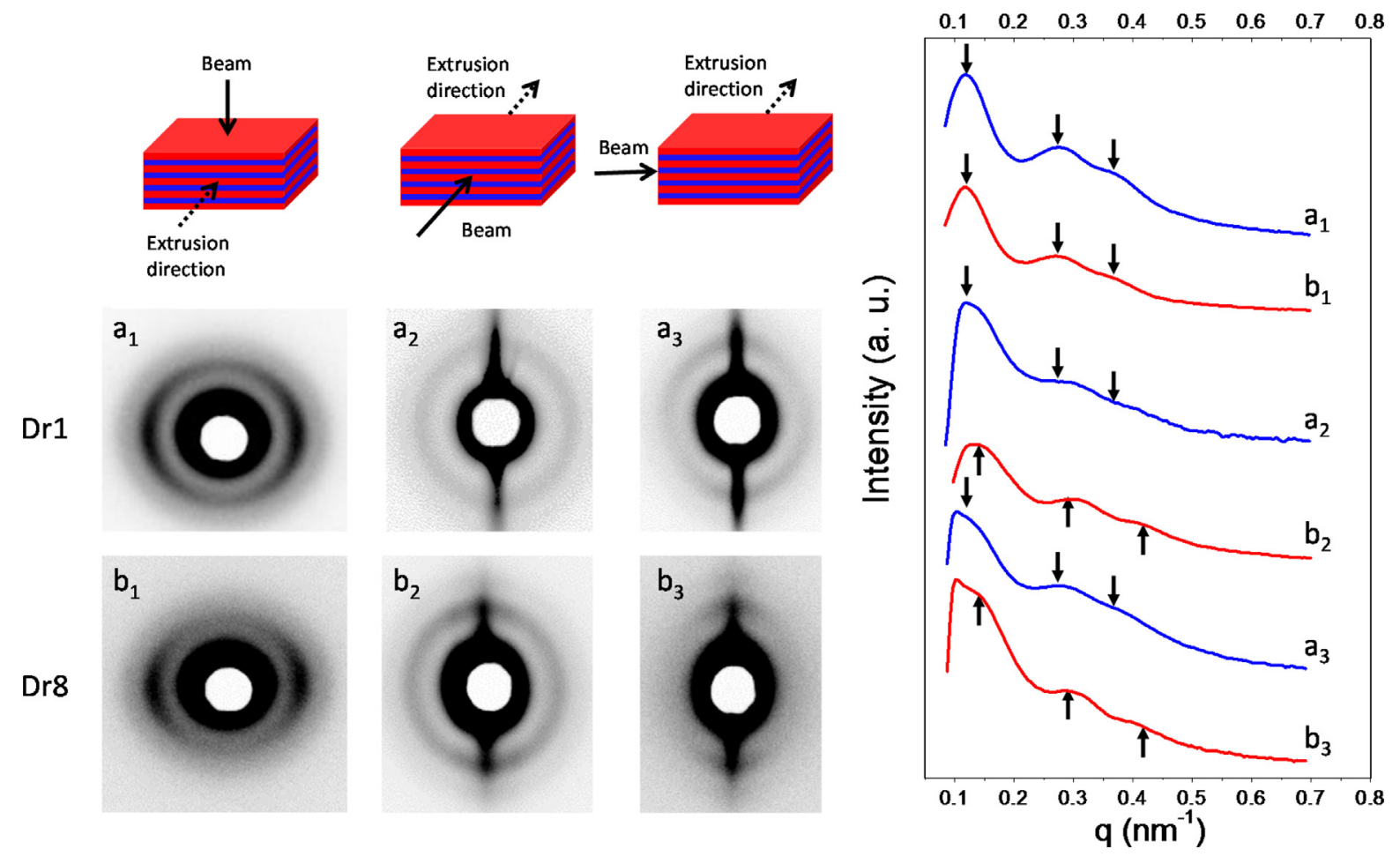

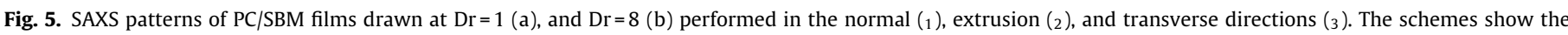

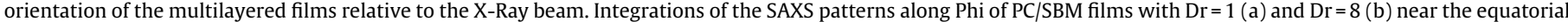

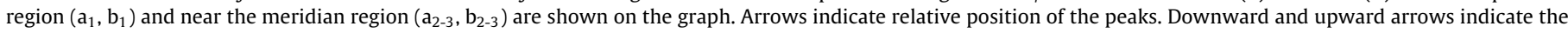
same position.

for PP/PS multilayered films [42] Additional annealing experiments were also performed at $145^{\circ} \mathrm{C}$ for 5 days (below the $T_{\mathrm{g}}$ of PC) to see if the ordering could be improved over time (Fig. SI-4). Contrary to what was observed by Burt et al. [10,12,43], SAXS patterns do not show any significant change, probably due to the lack of mobility of the copolymer at this temperature $\left(T_{\mathrm{g}, \mathrm{PMMA}}+15^{\circ} \mathrm{C}\right)$.

\section{Conclusions}

Poly(styrene-b-butadiene-b-methyl methacrylate) triblock copolymer has been successfully confined with polycarbonate through multilayer coextrusion. The resulting films have a total thickness between $100 \mu \mathrm{m}$ and $1 \mathrm{~mm}$ and are made of hundreds to thousands of alternating layers with the SBM layer thicknesses ranging from a few hundreds to a few tens of nanometers. We showed that despite performing extrusion below $\mathrm{T}_{\mathrm{ODT}}$ of the SBM, continuous self-assembled structures can be achieved without any further post-treatment such as annealing. We also evidenced, for the first time, long-range ordering for these "as extruded" materials, which, though imperfect, can be improved by increasing the draw ratio after extrusion and decreasing the SBM layer thickness. This industrially scalable and simple technique allows the continuous fabrication of hierarchically organized films that can be of interest for innovative mechanically-reinforced materials or other engineering applications where long-range ordering of the nanodomains is necessary for large quantities of material.

\section{Acknowledgments}

This work was funded by the CNAM through the project MATERAM. We would like to acknowledge Arkema for supplying the SBM; Dr M. Barbazanges and the Institut Parisien de Chimie Moléculaire, UMR CNRS 8232 for their help with the NMR characterizations; F. Portail, from Arkema GRL Lacq for her help with the sample preparation for TEM experiments; The Institut de Biologie Paris Seine IBPS/FR 3631 for providing access to its TEM, and G. Toutirais for her guidance with the apparatus; V. Michel for his help with the SAXS experiments; J-S. Montana for the analysis of the GPC curves, and A. Grandmontagne for the coextrusion of the samples. G. Miquelard-Garnier was appointed Editor for Materials Today Communications over the course of the paper submission process but he neither handled the manuscript nor was involved in the final decision.

\section{Appendix A. Supplementary data}

Supplementary data associated with this article can be found, in the online version, at http://dx.doi.org/10.1016/j.mtcomm.2015. 11.003.

\section{References}

[1] I.W. Hamley, Prog. Polym. Sci. 34 (2009) 1161-1210.

[2] H.-C. Kim, S.-M. Park, W.D. Hinsberg, Chem. Rev. Wash. DC U. S. 110 (2009) $146-177$.

[3] J.K. Kim, S.Y. Yang, Y. Lee, Y. Kim, Prog. Polym. Sci. 35 (2010) 1325-1349.

[4] A.P. Marencic, R.A. Register, Annu. Rev. Chem. Biomol. Eng. 1 (2010) 277-297.

[5] J. Bang, U. Jeong, D.Y. Ryu, T.P. Russell, C.J. Hawker, Adv. Mater. 21 (2009) 4769-4792.

[6] J.M. Carr, D.S. Langhe, M.T. Ponting, A. Hiltner, E. Baer, J. Mater. Res. 27 (2012) $1326-1350$

[7] M. Ponting, T.M. Burt, L.T.J. Korley, J. Andrews, E. Hiltner, A. Baer, Ind. Eng. Chem. Res. 49 (2010) 12111-12118.

[8] M. Ponting, E. Hiltner, A. Baer, Macromol. Symp. 294 (2010) 19-32.

[9] R.Y.F. Liu, Y. Jin, E. Hiltner, A. Baer, Macromol. Rapid Commun. 24 (2003) 943-948.

[10] T.M. Burt, J. Keum, A. Hiltner, E. Baer, L.T.J. Korley, ACS Appl. Mater. Interfaces 3 (2011) 4804-4811.

[11] T.M. Burt, A.M. Jordan, L.T.J. Korley, ACS Appl. Mater. Interfaces 4 (2012) 5155-5161.

[12] T.M. Burt, A.M. Jordan, L.T.J. Korley, Macromol. Chem. Phys. 214 (2013) $873-881$.

[13] F.S. Bates, G.H. Fredrickson, Phys. Today 52 (1999) 32-38. 
[14] V. Abetz, P.F.W. Simon, in: V. Abetz (Ed.), Block Copolymers I, vol. 189, Springer-Verlag, Berlin/Heidelberg, 2005, pp. 125-212.

[15] N. Hadjichristidis, H. Iatrou, M. Pitsikalis, A. Pispas, S. Avgeropoulos, Prog. Polym. Sci. 30 (2005) 725-782.

[16] W. Li, F. Qiu, A.-C. Shi, Macromolecules 45 (2011) 503-509.

[17] W. Zheng, Z.-G. Wang, Macromolecules 28 (1995) 7215-7223.

[18] P. Tang, F. Qiu, H. Zhang, Y. Yang, Phys. Rev. E (2004) 69.

[19] R. Stadler, C. Auschra, J. Beckmann, U. Krappe, I. Voight-Martin, L. Leibler Macromolecules 28 (1995) 3080-3097.

[20] W. Stocker, J. Beckmann, R. Stadler, J.P. Rabe, Macromolecules 29 (1996) $7502-7507$

[21] U. Breiner, U. Krappe, E.L. Thomas, R. Stadler, Macromolecules 31 (1998) $135-141$.

[22] U. Breiner, U. Krappe, T. Jakob, R. Abetz, V. Stadler, Polym. Bull. 40 (1998) 219-226.

[23] R. Vitali, E. Montani, Polymer 21 (1980) 1220-1222.

[24] J.H. Rosedale, F.S. Bates, Macromolecules 23 (1990) 2329-2338.

[25] C.D. Han, J. Kim, J.K. Kim, Macromolecules 22 (1989) 383-394.

[26] C.D. Han, D.M. Baek, J.K. Kim, Macromolecules 23 (1990) 561-570.

[27] C.D. Han, D.M. Baek, J.K. Kim, T. Hashimoto, S. Okamoto, Macromolecules 24 (1991) 5408-5413.

[28] C.D. Han, D.M. Baek, J.K. Kim, Macromolecules 28 (1995) 5886-5896.

[29] M. Rubinstein, S.P. Obukhov, Macromolecules 26 (1993) 1740-1750.

[30] E. Di Cola, C. Fleury, P. Panine, M. Cloitre, Macromolecules 41 (2008) 3627-3635
[31] P.J. Harris, J. Patz, B.A. Huntington, R.T. Bonnecaze, J. Meltzer, D. Maia, Polym. Eng. Sci. 54 (2014) 636-645.

[32] T. Abetz, V. Goldacker, Macromol. Rapid Commun. 21 (2000) 16-34.

[33] T. Goldacker, V. Abetz, Macromolecules 32 (1999) 5165-5167.

[34] R. Utracki, L.A. Simha, Polym. Int. 53 (2004) 279-286.

[35] D.W. van Krevelen, K. te Nijenhuis, Properties of Polymers Their Correlation with Chemical Structure; Their Numerical Estimation and Prediction from Additive Group Contributions, Elsevier, Amsterdam Boston, 2009.

[36] J. Brandrup, E.H. Immergut, E.A. Grulke, Polymer Handbook, 4th ed., Wiley New York; Chichester, 2004.

[37] I. Tokarev, R. Krenek, Y. Burkov, D. Schmeisser, A. Sidorenko, S. Minko, M. Stamm, Macromolecules 38 (2005) 507-516.

[38] S. Roland, D. Gaspard, R.E. Prud'homme, C.G. Bazuin, Macromolecules 45 (2012) 5463-5476.

[39] H. Hückstädt, A. Göpfert, V. Abetz, Polymer 41 (2000) 9089-9094.

[40] E.M. McCready, W.R. Burghardt, Macromolecules 48 (2015) 264-271.

[41] R. Mao, E.M. McCready, W.R. Burghardt, Soft Matter 10 (2014) 6198-6207.

[42] Y. Jin, M. Rogunova, A. Hiltner, E. Baer, R. Nowacki, A. Galeski, E. Piorkowska, J. Polym. Sci. Part B Polym. Phys. 42 (2004) 3380-3396.

[43] T.M. Burt, S. Monemian, A.M. Jordan, L.T.J. Korley, Soft Matter 9 (2013) 4381-4385. 